\title{
An indication of major genes affecting hip and elbow dysplasia in four Finnish dog populations
}

\author{
$\mathrm{K} \mathrm{Mäki}^{1}$, LLG Janss ${ }^{2}$, AF Groen ${ }^{3}$, A-E Liinamo ${ }^{3}$ and M Ojala ${ }^{1}$ \\ ${ }^{1}$ Department of Animal Science, PO Box 28, 00014 Helsinki University, Finland; 2ID-Lelystad Institute for Animal Science and Health, \\ PO Box 65, 8200 AB Lelystad, The Netherlands; ${ }^{3}$ Animal Breeding and Genetics Group, Wageningen University, PO Box 338, $6700 \mathrm{AH}$ \\ Wageningen, The Netherlands
}

\begin{abstract}
The aim of the study was to assess the possible existence of major genes influencing hip and elbow dysplasia in four dog populations. A Bayesian segregation analysis was performed separately on each population. In total, 34140 dogs were included in the data set. Data were analysed with both a polygenic and a mixed inheritance model. Polygenic models included fixed and random environmental effects and additive genetic effects. To apply mixed inheritance models, the effect of a major gene was added to the polygenic models. The major gene was modelled as an autosomal biallelic locus with Mendelian transmission probabilities. Gibbs sampling and a Monte Carlo Markov Chain algorithm were used. The goodness-of-fit of the different models were compared using the residual sum-of-squares. The existence of a major gene was considered likely for hip dysplasia in all
\end{abstract}

the breeds and for elbow dysplasia in one breed. Several procedures were followed to exclude the possible false detection of major genes based on non-normality of data: permuted datasets were analysed, data-transformations were applied, and residuals were judged for normality. Allelic effects at the major gene locus showed nearly to complete dominance, with a recessive, unfavourable allele in both traits. Relatively high estimates of the frequencies of unfavourable alleles in each breed suggest that considerable genetic progress would be possible by selection against major genes. However, the major genes that are possibly affecting hip and elbow dysplasia in these populations will require further study.

Heredity (2004) 92, 402-408, advance online publication, 3 March 2004; doi:10.1038/sj.hdy.6800434

Keywords: breeding; dog; elbow dysplasia; hip dysplasia; major gene

\section{Introduction}

Canine hip and elbow dysplasia are hereditary malformations that develop during periods of rapid growth. Each of these dysplasias can cause arthritis and/or severely deformed joints, disabling the dog. Hip and elbow dysplasia have been reported as quantitative traits occurring in numerous breeds (Leighton et al, 1977, Swenson et al, 1997, Mäki et al, 2002). In addition to quantitative inheritance, the possibility of a major gene (Todhunter et al, 1999, Everts, 2000) and of mitochondrial (Janss and Brascamp, 1998) inheritance have been suggested.

In a previous study (Mäki et al, 2002), the mode of inheritance of hip and elbow dysplasia was studied in four Finnish dog populations: the German Shepherd, the Golden Retriever, the Labrador Retriever, and the Rottweiler. The existence of segregating major genes was preliminarily assessed based on frequency distributions of hip and elbow dysplasia in the offspring of individual sires. These family-specific distributions did not show any signs of major genes, for example multimodality. This does not, however, rule out the possibility of a major gene affecting either of the traits, because the effect of a major gene would have to be very large to

Correspondence: K Mäki, Department of Animal Science, PO Box 28, 00014 FIN, Helsinki University, Finland.

E-mail: katariina.maki@animal.helsinki.fi

Received 3 February 2003; accepted 6 January 2004; published online 3 March 2004 affect the distributions of the traits in studies of this kind. Knowledge of the appropriate genetic model for hip and elbow dysplasia, and especially the possibility of being able to identify accurately carriers of deleterious alleles, would enhance the possibilities for setting up efficient selection and mating schemes.

The aim of this study was to perform a segregation analysis in order to assess the possible existence of major genes affecting hip and/or elbow dysplasia in four Finnish dog populations.

\section{Materials and methods}

\section{Materials}

Pedigree information and official radiographic examination results of 34140 dogs examined for hip and elbow dysplasia in the period of 1988-2000 were obtained from the Finnish Kennel Club. All the dogs had a record for hip dysplasia, but not necessarily one for elbow dysplasia. The breeds included in the study were the German Shepherd, the Golden Retriever, the Labrador Retriever, and the Rottweiler. Each dog had been given a separate grade for its right and its left joint. The mean of these grades was used as the trait studied.

The data of the Finnish Kennel Club can be considered to be a representative sample of these populations. In the four breeds included in this study, examination of the hip and elbow dysplasia status of breeding dogs is required for puppy registration. Also many voluntary examinations for other dogs in addition to breeding candidates 
are carried out. However, to some extent, examination results for the most severely affected dogs may not exist in the data, in particular with regard to elbow dysplasia. Very severe cases of elbow dysplasia will typically be operated on at a young age, before the official screening age of 1 year. The records of these dogs are not included in the database of the Finnish Kennel Club.

The hip dysplasia classification protocol changed twice during the recording period. The resulting three sets of hip grades were combined in this study to form classes 1 and 1.5 (normal hips), 2 (borderline dysplasia), 3 (mild dysplasia), 4 (moderate dysplasia), and 5.0, 5.5, and 6.0 (severe dysplasia). Classes 1.5, 5.0, and 6.0 were found only in old records, while, in the other classes, both old records and new records graded according to the current system existed. Elbow dysplasia consists of four separate growth disorders of the elbow joint: elbow incongruity, fragmented coronoid process, osteochondrosis dissecans, and ununited anconeal process. In Finland, these different growth disorders are not separated from each other when grading the joints. In the data, elbow dysplasia was classified according to the International Elbow Working Group protocol, from normal or borderline (0) to mild (1), moderate (2), or severe (3) dysplasia. The classification was based on the existence and severity of arthritic changes on the joint surfaces.

The pedigrees of the dogs analysed were quite effectively linked together. It is common in dog populations to have a few popular sires, which sire a large part of the offspring born in a given year. These sires form links between different lines. The pedigrees used in this study covered all the dogs born from 1978 to 2000. A large part of the dogs that were born during 1972-1977, and some dogs born before 1972 were also included.

\section{Genetic models}

A polygenic animal model, assuming additive genetic effects, was first applied for each breed. An effect of a major gene genotype was added to the polygenic models to analyse the existence of a major gene. The complete mixed inheritance model was

$$
\mathbf{y}=\mathbf{X} \mathbf{1} \boldsymbol{\beta}+\mathbf{X} 2 \mathbf{b}+\mathbf{X} 3 \mathbf{c}+\mathbf{Z u}+\mathbf{Z W m}+\mathbf{e},
$$

where $\mathbf{y}=$ grades for hip dysplasia or elbow dysplasia; mean of the right and left joint, $\boldsymbol{\beta}=$ nongenetic fixed effects, $\mathbf{b}=$ random breeder effects, $\mathbf{c}=$ random litter effects, $\mathbf{u}=$ additive genetic effects, $\mathbf{W}=$ genotypes, $\mathbf{m}=$ genotype means, and $\mathbf{e}=$ random residuals. $\mathbf{X} \mathbf{1}, \mathbf{X} \mathbf{2}$, $\mathbf{X} 3$, and $\mathbf{Z}$ were incidence matrices relating nongenetic, breeder, litter, and genetic effects, respectively, to the observations. The nongenetic effects included effects of age, sex, year of birth, and of the radiologist grading the X-rays. The distributions of $\mathbf{b}, \mathbf{c}, \mathbf{u}$, and $\mathbf{e}$ were assumed to be $\mathbf{b} \sim N\left(0, \mathbf{I} \sigma_{b}^{2}\right), \mathbf{c} \sim N\left(0, \mathbf{I} \sigma_{c}^{2}\right), \mathbf{u} \sim N\left(0, \mathbf{A} \sigma_{u}^{2}\right)$ and e $\sim N\left(0, \mathbf{I} \sigma_{e}^{2}\right)$, where $A$ is the numerator relationship matrix. Variances were conditional about the fixed effects and the effects associated with the major gene. Flat prior distributions were defined for the nongenetic effects and effects at the single locus $(-\infty, \infty)$, for the variance components $(0, \infty)$, and for the allele frequencies $(0,1)$. By using flat priors, the posterior density investigated is mathematically equal to the likelihood and in that sense, compared to likelihood-based inference, the priors are not expected to introduce bias. Variances were assumed, a priori, to be positive; the prior was defined
Major genes for canine hip and elbow dysplasia

on $\left[10^{-12}, \infty\right]$. The major gene was modelled as an autosomal biallelic locus with Mendelian transmission probabilities of the genotypes: 1.0 for A1A1, 0.5 for A1A2, and 0.0 for A2A2 to transmit the allele A1. The allele that decreases the value of the phenotypic measurement (ie, the favourable allele) was defined as A1, and the allele that increases it (ie, the unfavourable allele) as A2. $\mathbf{W}$ was a four-column matrix indicating the genotype of each animal: A1A1, A1A2, A2A1, or A2A2. Each column of the matrix was a dummy variable, the elements of which were coded 1 if the given animal had that genotype and 0 otherwise. Effects of the genotypes were in $\mathbf{m}$, with $\mathbf{m}^{\prime}=(-a, d, d, a)$, where $a$ was referred to as the additive and $d$ as the dominant effect at the major gene locus.

Goodness-of-fit of the polygenic and major gene models were compared using the residual sums-ofsquares (SS) as in Palmer et al. (2001).

\section{Deviations from normal distribution}

Segregation analysis may be sensitive to deviations from normality of the trait distribution, when such deviations are not caused by a single gene (Lynch and Walsh, 1998). In this study, the within-breed distributions of hip and elbow dysplasia grades were not normal, because of the combined grades of all three classification systems. In addition, taking the mean of the left and right joints as the studied trait resulted in the distributions being even more complicated. On the other hand, taking the mean caused the trait units to be more continuous, making it appropriate to analyse hip and elbow dysplasia as continuous traits. As a result, altogether 11-15 possible values existed in hip dysplasia, and likewise seven possible values in grades of elbow dysplasia (Figures 1 and 2). This resulted from the possibility of the left and the right joint having different grades of dysplasia. The distributions were multimodal for hip dysplasia in all the breeds and skewed for elbow dysplasia in all breeds except the Rottweiler, which had a bimodal distribution.

Although the phenotypic distributions deviated from normal in all breeds and both traits, it is possible that the distribution of residuals is not very different from normal after fitting statistical models. Standard residual

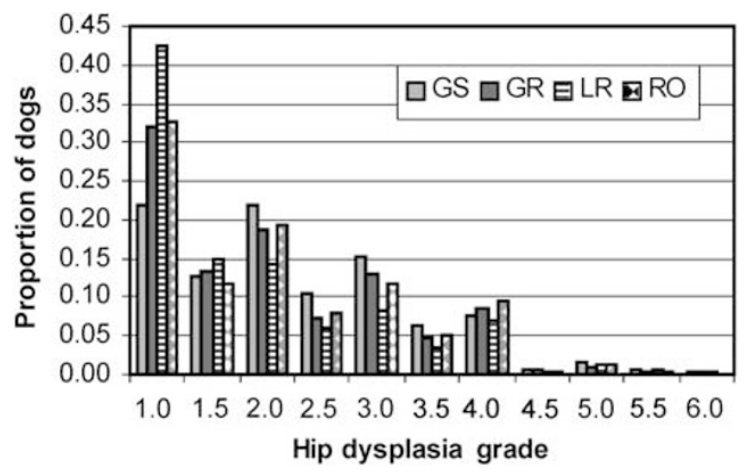

Figure 1 Distribution of the dogs studied based on hip dysplasia grades; mean of the left and the right joint. Only 11 values are shown since the other four were so rare that their frequency was lower than $0.5 \%$. GS = German Shepherd, $N=13501 ; \mathrm{GR}=$ Golden Retriever, $\quad N=8440 ; \quad \mathrm{LR}=$ Labrador Retriever, $\quad N=7820$; $\mathrm{RO}=$ Rottweiler,$\quad N=4379 . \quad 1.0=$ normal,$\quad 2.0=$ borderline, $3.0=$ mild, $4.0=$ moderate, $5.0-6.0=$ severe dysplasia. 


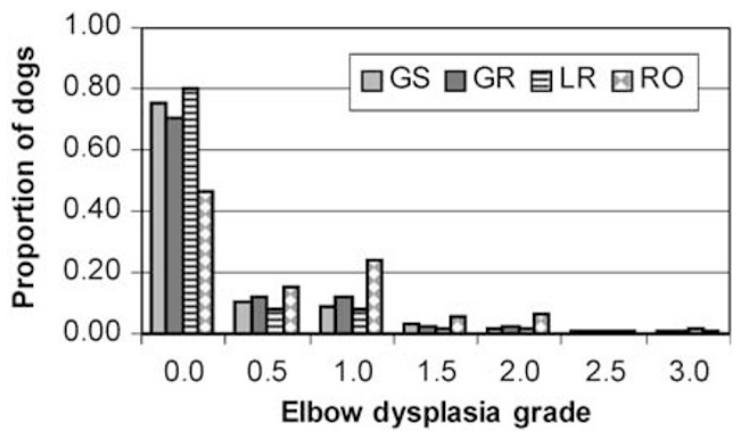

Figure 2 Distribution of dogs based on elbow dysplasia grades; mean of the left and the right joint. GS =German Shepherd, $N=3542 ; \mathrm{GR}=$ Golden Retriever, $N=2642 ; \mathrm{LR}=$ Labrador Retriever, $N=2627 ; \mathrm{RO}=$ Rottweiler, $N=3386.0 .0=$ normal or borderline, $1.0=$ mild, $2.0=$ moderate, $3.0=$ severe dysplasia.

analyses and Box-Cox normality plots were made for the polygenic models, to study normality of the residuals. For the elbow dysplasia models of the German Shepherd, the Golden Retriever, and the Labrador Retriever, a clear deviation from normality was observed. The Box-Cox normality plot suggested an inversion transformation for all the three cases. The transformations did not change the phenotypic distributions much, but they indeed changed the distribution of the residuals towards normal.

The mixed inheritance analyses of elbow dysplasia in these breeds were made from both the 'raw' and the transformed data. The transformed trait was given the name EDT (elbow dysplasia transformed), while the 'raw' traits were called ED (elbow dysplasia) and HD (hip dysplasia). It was assumed that if the data sets had a normal residual distribution when fitting the polygenic models, normality was maintained when the models were extended to include the major gene effects.

\section{Computing procedures}

Detection of major genes was based on a Bayesian modelling approach to segregation analysis (Janss et al, 1995). Computations were performed using Gibbs sampling and the Monte Carlo Markov Chain (MCMC) algorithm with the program MaGGic (Janss, 1998). The application of MCMC techniques was found to be required here, because deterministic approaches, such as peeling, are intractable on typical animal populations, which have many loops due to inbreeding and multiple matings. However, also in the choice of MCMC techniques, care has to be taken because simple (scalar) Gibbs sampling algorithms 'get stuck' on pedigrees with large families, especially when estimating genotype effects. This can result to slow mixing of genotype states, and consequently, in convergence problems (Janss et al, 1995). The program MaGGic used here was especially developed for animal breeding data, and implements a blockwise sampling of each sire and its final progeny, which greatly improves mixing and convergence. The blockwise sampling can also be seen as 'clipping' of the pedigree, because the algorithms involve the removal of final progeny by deterministic peeling before sampling of a sire's genotype. Although mixing is largely improved with the blocking technique (Janss et al, 1995), proper convergence of MCMC algorithms still requires scrutiny, which can effectively be done by comparing results from multiple medium-long chains.

For polygenic models, five chains were run per trait, each consisting of 25500 cycles including a burn-in period of 500 cycles. This number of cycles was sufficient to allow convergence of the model components. Samples of model parameters were written out every 50th cycle, resulting in 500 samples per chain.

Five chains per trait were run also for the mixed inheritance models. For the German Shepherd, the Golden Retriever, and the Rottweiler, chains were each of 40500 cycles. The HD and ED data sets in the Labrador Retriever reached convergence more slowly, and 100000 additional cycles were run for each chain. From these cycles, samples of model parameters were written out every 100th cycle, resulting in 1000 samples per chain.

A model relaxation technique (Sheehan and Thomas, 1993) was used to improve convergence of the Gibbs sampler for the major gene component in the mixed models. This method involves relaxation of the transmission probabilities to slightly non-Mendelian by the use of $\tau_{\mathrm{A} 1, \mathrm{~A} 1 \mathrm{~A} 1}=1-p_{\text {rel }}$ and $\tau_{\mathrm{A} 1, \mathrm{~A} 2 \mathrm{~A} 2}=p_{\text {rel }}$, where $\tau_{\mathrm{A} 1, \mathrm{~A} 1 \mathrm{~A} 1}$ is the transmission probability for genotype $\mathrm{A} 1 \mathrm{~A} 1$ and $\tau_{\mathrm{A} 1, \mathrm{~A} 2 \mathrm{~A} 2}$ is the transmission probability for genotype A2A2, in each case of transmitting the A1 allele, and $p_{\text {rel }}$ is a small probability for 'non-Mendelian transmission'. For inference about the Mendelian model, only cycles with a Mendelian genotype configuration were used. To achieve a desired rate of Mendelian samples, trial runs were run to determine a suitable relaxation factor $\left(p_{\text {rel }}\right)$. For each data set, a target rate of Mendelian samples of $2-5 \%$ was set.

Evaluation of the burn-in period and computing of the mean, mode and standard deviation of the posterior distribution were carried out with the program Gibanal (van Kaam, 1998). Convergence of the Gibbs sampler was evaluated from multiple chains with different seeds by plotting a parameter value versus cycle number. For the Labrador data of HD and ED, the significance of the chain effect was also analysed by ANOVA where no significant chain differences should be found if convergence is reached and if subsequent samples from the chain are indeed independent. Nonparametric estimates of posterior marginal densities were computed with Gibanal (van Kaam, 1998).

Major gene variance $\left(\sigma_{w}^{2}\right)$ was computed from the genotype frequencies and the genotype effects $a$ and $d$ in each Gibbs sample as $2 p q(a+d(q-p))^{2}+(2 p q d)^{2}$, where $p$ is the frequency of the unfavourable A2 allele and $q=1-p$ (Falconer and Mackay, 1996). Nonsignificance of a variance component has been shown to lead to a posterior distribution with a global mode of zero (Janss et al, 1995), whereas significance of the variance component shows a global mode greater than zero. A more conservative criterion was applied in this study, the estimate of $\sigma_{w}^{2}$ was accepted to be significant only when the global mode for $\sigma_{w}^{2}>0$ had a 20-fold larger density compared to the density at $\sigma_{w}^{2}=0$.

\section{Reducing the possibility of false positives}

Further, reanalyses from randomly drawn samples were made to evaluate the possibility of false detection of major genes. Randomly drawn samples were made by 
shuffling, that is, each trait value and the corresponding model effects were reassigned to a new individual, while the individual's genetic links were retained (Churchill and Doerge, 1994). If the detection of a major gene were caused by for example environmental factors, shuffling the data would not affect the estimate of the major gene variance. On the other hand, if detection of a major gene were 'real', shuffling the data should obscure the detection of a major gene.

Five shuffled sets for each data where a major gene had been detected were created, and each shuffled data set was analysed by a single chain of 40500 cycles (100000 for the Labrador Retriever). If a major gene variance was detected from an analysis of a shuffled data set, the hypothesis of no major gene $\left(H_{0}\right)$ was accepted. If any analyses of the five shuffled data sets did not give similar estimates of a major gene as the analyses from the original data, $H_{0}$ was rejected, and the existence of a major gene was considered as likely.

\section{Results}

\section{Convergence}

In general, convergence was good in every breed and trait, except for the Labrador Retriever data sets HD and ED, with which some problems occurred. With the Labrador Retriever data, distinct periods were detected in the chains when using relaxation: in some periods there were numerous samples with Mendelian genotype configuration, while in others, even long ones, no Mendelian samples were achieved at all. Owing to this, longer chains without relaxation were run for that data set. This resulted in some chains converging well, also according to the F-statistics of ANOVA, while, in other chains, the estimates stayed at a given level until almost the end of chain was reached, when they jumped to a totally new level. Estimates of the parameters were calculated only from the chains that had reached adequate convergence.

\section{Variance components}

For HD and ED, statistically significant major gene variance was detected in each breed (Figures 3 and 4). Comparing the HD and ED polygenic model with the mixed inheritance model, polygenic variation $\left(\sigma_{u}^{2}\right)$ was drastically smaller in the latter model in all studied cases. In the German Shepherd, HD polygenic variance as a proportion of phenotypic variance changed the least

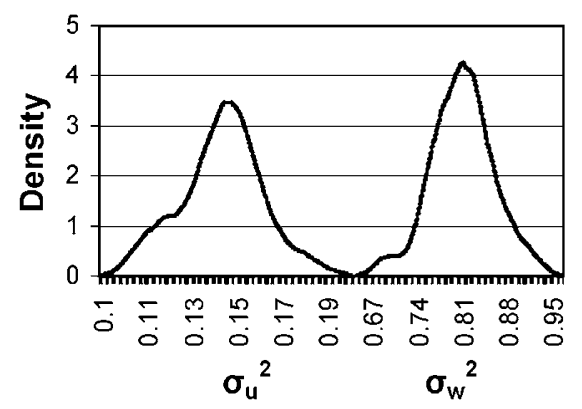

Figure 3 Estimated nonparametric marginal densities for polygenic $\left(\sigma^{2} u\right)$ and major gene $\left(\sigma^{2} w\right)$ variance for hip dysplasia (HD) in the German Shepherd, as an example of all breeds.

compared to other breeds, that is, from 0.23 to 0.10 (Table 1), and in the Rottweiler it changed the most, that is, from 0.36 to 0.11 (Table 4). For ED, this change was smallest in the Labrador Retriever, that is, from 0.13 to 0.01 (Table 3), and largest in the Rottweiler, namely from 0.38 to 0.11 . The estimate of polygenic variance from the mixed inheritance models still remained statistically significant for HD in each breed, while, for ED, it stayed significant only in the Rottweiler. No statistically significant major gene variances for EDT were detected in any of the three breeds.

The mixed inheritance models had a larger residual SS than the polygenic models in both traits and all breeds. The percentage change in the residual SS relative to polygenic model varied from 40 in the German Shepherd to 66 in the Labrador Retriever for HD, and from 41 in the Rottweiler to 72 in the Labrador Retriever for ED.

\section{Allele effects and frequencies}

Allele effects at the major gene revealed close to complete dominance for HD and ED in each breed, and possibly a slight overdominance for HD in the German Shepherd (Tables 1-4). The favourable allele A1 was dominant in every breed, based on the negative estimates of the dominance effect $d$ at the major gene. A negative dominance value means that the heterozygote is more similar to the low, favourable homozygote (A1A1) than to the high, affected homozygote (A2A2). Recall that a low estimate on these scales means better joints, as they correspond to a smaller degree of dysplasia.

The estimates of allele effects were quite large. The difference between the extreme homozygous genotypes $(=2 a)$ was approximately two phenotypic standard deviations for both traits, and in all breeds except the Labrador Retriever. In this breed, the difference between the extreme genotypes in ED was even larger, that is, approximately four times the phenotypic standard deviation.

The frequencies of the unfavourable allele A2 were high in all breeds, varying from 0.38 in the Rottweiler to 0.62 in the German Shepherd for HD, and from 0.58 in the Rottweiler to 0.78 in the Labrador Retriever for ED.

\section{Effect of the data shuffling}

In the German Shepherd, the Golden Retriever, and the Labrador Retriever, estimates of the major gene variance

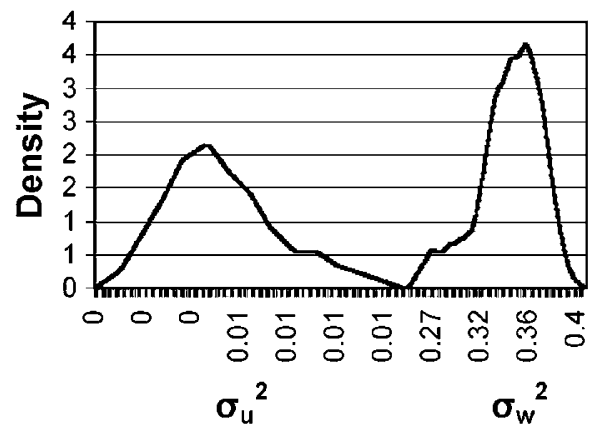

Figure 4 Estimated nonparametric marginal densities for polygenic $\left(\sigma^{2} u\right)$ and major gene $\left(\sigma^{2} w\right)$ variance for elbow dysplasia (ED) in the Golden Retriever, as an example of all breeds. 
Table 1 Estimates of marginal posterior means and standard deviations (in brackets) for phenotypic variance $\left(\sigma_{p}^{2}\right)$, proportion of additive $\left(h^{2}\right)$, major gene $\left(w^{2}\right)$, breeder $\left(b^{2}\right)$, and litter $\left(c^{2}\right)$ variance from the phenotypic variance, as well as for allele effects $(a$ and $d)$ and the frequency of the favourable A1 allele (Fr(A1)) for hip and elbow dysplasia in the German Shepherd

\begin{tabular}{lcccccccc}
\hline Trait and model & $\sigma_{p}^{2}$ & $h^{2}$ & $w^{2}$ & $b^{2}$ & $c^{2}$ & $a$ & $d$ \\
\hline HDPa & 1.11 & 0.23 & - & 0.06 & 0.06 & - & - \\
EDP & 0.28 & 0.18 & - & 0.07 & 0.11 & - & - \\
HDM & 1.48 & 0.10 & 0.54 & 0.03 & 0.03 & $0.85(0.03)$ & $-1.02(0.04)$ \\
EDM & 0.47 & 0.02 & 0.85 & 0.00 & 0.02 & $0.65(0.01)$ & $-0.64(0.01)$ & $0.62(0.03)$ \\
\hline
\end{tabular}

${ }^{a} \mathrm{HDP}, \mathrm{EDP}=$ hip and elbow dysplasia, polygenic model; HDM, EDM = hip and elbow dysplasia, mixed inheritance model.

$-=$ estimate not relevant in the model assumed.

Table 2 Estimates of marginal posterior means and standard deviations (in brackets) for phenotypic variance $\left(\sigma_{p}^{2}\right)$, proportion of additive $\left(h^{2}\right)$, major gene $\left(w^{2}\right)$, breeder $\left(b^{2}\right)$, and litter $\left(c^{2}\right)$ variance from the phenotypic variance, as well as for allele effects $(a$ and $d)$ and the frequency of the favourable A1 allele ( $\operatorname{Fr}(\mathrm{A} 1))$ for hip and elbow dysplasia in the Golden Retriever

\begin{tabular}{lcccccccc}
\hline Trait and model & $\sigma_{p}^{2}$ & $h^{2}$ & $w^{2}$ & $b^{2}$ & $c^{2}$ & $a$ & $d$ \\
\hline HDPa & 1.15 & 0.27 & - & 0.06 & 0.05 & - & - \\
EDP & 0.31 & 0.26 & - & 0.03 & 0.06 & - & - \\
HDM & 0.86 & 0.07 & 0.50 & 0.03 & 0.02 & $0.92(0.02)$ & $-0.91(0.04)$ \\
EDM & 0.44 & 0.02 & 0.80 & 0.00 & 0.00 & $0.63(0.01)$ & $-0.61(0.02)$ & $0.40(0.04)$ \\
\hline
\end{tabular}

${ }^{a} \mathrm{HDP}, \mathrm{EDP}=$ hip and elbow dysplasia, polygenic model; HDM, EDM = hip and elbow dysplasia, mixed inheritance model.

$-=$ estimate not relevant in the model assumed.

Table 3 Estimates of marginal posterior means and standard deviations (in brackets) for phenotypic variance $\left(\sigma_{p}^{2}\right)$, proportion of additive $\left(h^{2}\right)$, major gene $\left(w^{2}\right)$, breeder $\left(b^{2}\right)$, and litter $\left(c^{2}\right)$ variance from the phenotypic variance, as well as for allele effects $(a$ and $d)$ and the frequency of the favourable A1 allele ( $\operatorname{Fr}(\mathrm{A} 1))$ for hip and elbow dysplasia in the Labrador Retriever

\begin{tabular}{lcccccccc}
\hline Trait and model & $\sigma_{p}^{2}$ & $h^{2}$ & $w^{2}$ & $b^{2}$ & $c^{2}$ & $a$ & $d$ \\
\hline HDPa & 1.17 & 0.26 & - & 0.07 & 0.02 & - & - \\
EDP & 0.30 & 0.13 & - & 0.07 & 0.10 & - & - \\
HDM & 1.22 & 0.04 & 0.71 & 0.02 & 0.01 & $1.09(0.02)$ & $-1.04(0.02)$ \\
EDM & 1.03 & 0.01 & 0.89 & 0.00 & 0.03 & $0.96(0.02)$ & $-0.93(0.03)$ & $0.49(0.04)$ \\
\hline
\end{tabular}

${ }^{a} \mathrm{HDP}, \mathrm{EDP}=$ hip and elbow dysplasia, polygenic model; HDM, EDM = hip and elbow dysplasia, mixed inheritance model.

$-=$ estimate not relevant in the model assumed.

Table 4 Estimates of marginal posterior means and standard deviations (in brackets) for phenotypic variance $\left(\sigma_{p}^{2}\right)$, proportion of additive $\left(h^{2}\right)$, major gene $\left(w^{2}\right)$, breeder $\left(b^{2}\right)$, and litter $\left(c^{2}\right)$ variance from the phenotypic variance, as well as for allele effects $(a$ and $d)$ and the frequency of the favourable A1 allele ( $\operatorname{Fr}(\mathrm{A} 1))$ for hip and elbow dysplasia in the Rottweiler

\begin{tabular}{lccccccc}
\hline Trait and model & $\sigma_{p}^{2}$ & $h^{2}$ & $w^{2}$ & $b^{2}$ & $c^{2}$ & $a$ & $d$ \\
\hline HDPa & 1.21 & 0.36 & - & 0.07 & 0.04 & - & - \\
EDP & 0.48 & 0.38 & - & 0.02 & 0.04 & - & - \\
HDM & 0.90 & 0.11 & 0.50 & 0.03 & 0.03 & $0.91(0.04)$ & $-1.00(0.06)$ \\
EDM & 0.62 & 0.11 & 0.61 & 0.02 & 0.02 & $0.66(0.02)$ & $-0.64(0.04)$ \\
\hline
\end{tabular}

${ }^{a} \mathrm{HDP}, \mathrm{EDP}=$ hip and elbow dysplasia, polygenic model; HDM, EDM = hip and elbow dysplasia, mixed inheritance model.

$-=$ estimate not relevant in the model assumed.

for ED from the shuffled data sets were, at least in one data set, similar to the estimates obtained from the original data. These were the same data sets that were found to have a non-normal distribution of the residuals, and in which no major genes could be found when the trait values were transformed (EDT) in order to obtain a normal distribution for the residuals. For HD, no similar anomalous estimates were obtained in the shuffled data sets in any breed, and all the major gene variance estimates were between zero and 0.02 .

\section{Discussion}

\section{Variance components}

Estimates of major gene variance were high in both HD and ED in all breeds studied. Reduction of the additive genetic variance in the mixed inheritance models, when compared to the polygenic models, was very large. As a result of the remaining polygenic variation, the trait values can be considered to be close to continuous.

The estimates of the variance components suggest that the mode of inheritance of hip dysplasia is quantitative, 
with a major gene affecting the trait jointly with numerous minor genes. In elbow dysplasia, further analyses suggested the existence of major genes only in the Rottweiler. Previously, a possibility of the existence of a major gene for hip dysplasia has been reported in the German Shepherd Dog and the Labrador Retriever (Leighton, 1997). This finding was based on a major gene index (MGI; LeRoy and Elsen, 1992). In a crossbred data set of Labrador Retriever $\times$ Greyhound dogs, a linkage analysis was performed (Todhunter et al, 1999). It was found that at least two major genes were influencing hip dysplasia. The previous evidence for effects on elbow joints was more equivocal. Everts (2000) suggested a major gene model for fragmented coronoid process, which is one form of a growth disorder in the elbow joint, but approximately $80 \%$ of the dog genome was excluded as a candidate region in a search of markers, under a hypothesis of a recessive inheritance.

\section{Allele effects and frequencies}

The possible major genes were found to be recessive in each breed. Contrary to this finding, the two major genes reported to affect hip dysplasia in the previous Labrador Retriever $\times$ Greyhound crosses were dominant (Todhunter et al, 1999). However, in that study, hip dysplasia phenotypes were divided into different components, that is, joint laxity and dorsolateral subluxation, while, in this study, all components of the hip were considered jointly.

The relatively high estimates of the frequencies of the unfavourable recessive alleles in each breed, especially for hip dysplasia, suggest that it would be possible to gain considerable genetic progress by selection against a major gene. This could be done, for example, by selecting breeding dogs according to genotype probabilities estimated with the kind of a computer program used in this study. It is conceivable that genetic markers might be found to act as selection criteria in these populations. Phenotypic selection against both hip and elbow dysplasia has, thus far, been mainly ineffective in these populations: it has been based on small selection intensities and has resulted in very small or negligible genetic progress (Mäki et al, 2002). However, BLUP breeding values for hip and elbow dysplasia are being introduced for these populations. Genetic progress will be expected, as BLUP values are an effective tool for breeding.

When the allele frequencies are intermediate, as they are in most estimates reported in this study, the trait distribution with a major gene should be platykurtic, that is, flatter than normal (Lynch and Walsh, 1998). This was not observed, however. In a previous study (Mäki et al, 2002) on the same breeds and data sets, no indication of major genes was found based on within-sire phenotypic distributions of the left hip and elbow joints of the dogs.

\section{Effect of the trait distribution}

No major gene variances were obtained for HD from the shuffled data sets, and thus it can be considered likely that evidence of major genes was unlikely to have been a spurious result caused by nongenetic effects. Only five randomisations per trait and breed were carried out and more might be desirable. Nevertheless, other results also obtained in this study suggested the conclusion that there is a major gene for hip dysplasia.

Estimates of the same magnitude for ED major gene variance were obtained from both the original and shuffled data sets in the three breeds, excluding the Rottweiler. For the same three breeds, residuals were not normally distributed in the polygenic ED models. No major genes were detected from the transformed data sets (EDT) either. The results from the shuffled and the transformed data sets were interpreted as undermining the major gene theory for elbow dysplasia, although the mixed inheritance models provided a clearly better fit to the data than did the polygenic models for both HD and ED. In addition, other results from this study seem not to support the major gene theory for elbow dysplasia in the three breeds. The very large estimates of major gene variance and allele frequency for ED in all breeds, and especially in the Labrador Retriever, seem to be overestimations when considered together with all the other results. Moreover, convergence was difficult to reach for the mixed inheritance model in the Labrador Retriever data; this could be an indication of a nonfit to the model. However, it is possible that if the different disorders included in the term 'elbow dysplasia' were separated in the data of the Finnish Kennel Club, a separate study could find major genes. The study of Everts (2000) supports this hypothesis, having suggested a major gene for fragmented coronoid process.

Major genes that may affect hip and elbow dysplasia in these dog populations would repay further study. The data would be more informative if the clinical data of the worst cases, that is, dogs with operated joints, could be included. Acquiring more reliable information about the existence of the possible major genes would require, for example, a planned mating or crossing experiment between suitable parents or breeds.

\section{Acknowledgements}

The Finnish Kennel Club is acknowledged for providing the data for this study. It also financed the study, in part, with the Emil Aaltonen Foundation and the breed clubs for the Finnish Hound, the Rottweiler, and the German Shepherd. Veijo Vilva is acknowledged for providing help with various analyses.

\section{References}

Churchill GA, Doerge RW (1994). Empirical threshold values for quantitative trait mapping. Genetics 138: 963-971.

Everts RE (2000). Molecular Genetic Studies in the Dog; Application to Fragmented Coronoid Process (FCP) in the Labrador Retriever. PhD Thesis,. University of Utrecht.

Falconer DS, Mackay TFC (1996). Introduction to Quantitative Genetics. 4th edn. Longman Group: Essex.

Janss LLG (1998). Maggic: a package of subroutines for genetic analyses with Gibbs sampling. Proceedings of the Sixth World Congress on Genetics Applied to Livestock Production: Armidale, Australia. Vol. 27, pp 459-460.

Janss LLG, Brascamp EW (1998). Prediction of elbow dysplasia in dogs by breeding values of relatives. Proceedings of the Sixth World Congress on Genetics Applied to Livestock Production: Armidale, Australia. Vol. 25, pp 621-624.

Janss LLG, Thompson R, Van Arendonk JAM (1995). Application of Gibbs sampling for inference in a mixed major gene - polygenic inheritance model in animal populations. Theor Appl Genet 91: 1137-1147.

Leighton EA (1997). Genetics of canine hip dysplasia. J Am Vet Med Assoc 210: 1474-1479. 
Leighton EA, Linn JM, Willham RL, Castleberry MW (1977) A genetic study of canine hip dysplasia. Am J Vet Res 38: 241-244.

LeRoy P, Elsen JM (1992). Simple test statistics for major gene detection: a numerical comparison. Theor Appl Genet 83: 635-644.

Lynch M, Walsh B (1998). Genetics and Analysis of Quantitative Traits. Sinauer: Sunderland, Massachusetts.

Mäki K, Groen AF, Liinamo A-E, Ojala M (2002). Genetic variances, trends and mode of inheritance for hip and elbow dysplasia in Finnish dog populations. Anim Sci 75: 197-207.

Palmer LJ, Cookson WOCM, James AL, Musk AW, Burton PR (2001). Gibbs sampling-based segregation analysis of asthmaassociated quantitative traits in a population-based sample of nuclear families. Gen Epidem 20: 356-372.
Sheehan N, Thomas A (1993). On the irreducibility of a Markov chain defined on a space of genotype configurations by a sampling scheme. Biometrics 49: 163-175.

Swenson L, Audell L, Hedhammar ^ (1997). Prevalence and inheritance of and selection for elbow arthrosis in Bernese mountain dogs and Rottweilers in Sweden and benefit:cost analysis of a screening and control program. J Am Vet Med Assoc 210: 215-221.

Todhunter RJ, Acland GM, Olivier M, Williams AJ, VernierSinger M, Burton-Wurster N (1999). An outcrossed canine pedigree for linkage analysis of hip dysplasia. J Heredity $\mathbf{9 0}$ 83-92.

van Kaam JBCHM (1998). GIBANAL. Analysing Program for Monte Carlo Markov Chain Sequences. Release 2.7. University of Wageningen, Wageningen. 